\title{
B01 | Requirements of Petroelastic Models in Reservoir Characterization for Flow Simulation
}

\author{
By Don Caldwell (Marathon Oil)
}

\section{Introduction}

Rock physics is a key component of any quantitative seismic data integration within the reservoir simulation workflow. It connects the petrophysical properties of the rocks and fluids to the seismic response. A petroelastic model, PEM, is a forward set of rules that act as a link between the reservoir and elastic domains.

Scale is important to the PEM definition. In order to facilitated data integration into flow models, PEMs must be generated and implemented at flow scale. Often, PEMs are defined and calibrated for each lithology type at core and log scale which is usually smaller than the flow regime. These "calibrated" PEMs are also significantly smaller than the resolution of the seismic data used to constrain the reservoir property estimation. Up-scaling the PEM response is often required. If a PEM existed for each heterogeneous rock and fluid combination in the reservoir, the number of PEMs would be unmanageable. Therefore a workflow is implemented to predict the minimum number of PEMs required to describe the observed seismic response for given number reservoir flow layers. The workflow essentially optimizes the a number of PEM's at different scales to achieve an acceptable well synthetic to seismic tie with correct a porosity prediction.

It is important to define the PEM as a function of the significant properties that might vary within the reservoir during production. There are numerous rock physics models and mathematical relationships that can be employed to describe the elastic behaviour of the reservoir rock and fluid system. Variable lithologies, overburden stress, compaction, fluid type, pressure, all contribute to the seismic response. For example, grain density and modulus combined to construct the porous rock matrix, while fluid type, density, and moduli combine to determine the elastic properties of the pore filling fluids. These are then used to calculate the density, shear and bulk moduli, and the ensuing velocity of the flow scaled reservoir. From the velocity and density, acoustic and shear impedance is obtained to link the seismic response.

Geologically-consistent assignment of the PEM in the reservoir modelling is important. Rock and fluid properties vary spatially and temporally throughout the reservoir and PEMs created from well data may not adequately sample the gamut of rock and fluids within the reservoir. Geological models to predict locations of texturally-controlled PEMs and fluid distributions then become important to the model construction. Sub-seismic depositional influence on grain size and sorting, diagenetic effects, and lithologic variations require alternative PEM assignments consistent with the 3-D geologic volume.

Lastly, software and workflows need to take advantage of the PEMs. As the link between the reservoir and the elastic properties, the PEM can forward model the seismic response as well as reverse predict the reservoir properties from the seismic data. Marathon and CCGV jointly developed software to implement these concepts on any number of 3-D seismic volumes. 